\title{
ON ABSOLUTE SUMMABILITY FACTORS
}

\author{
HÜSEYIN BOR
}

(Communicated by Andrew M. Bruckner)

\begin{abstract}
In this paper a theorem on $\left|\bar{N}, p_{n}\right|_{k}$ summability factors, which generalizes a theorem of Mazhar (Indian J. Math. 14 (1972), 45-48) on $|C, 1|_{k}$ summability factors, has been proved.
\end{abstract}

\section{INTRODUCTION}

Let $\sum a_{n}$ be a given infinite series with partial sums $\left(s_{n}\right)$. We denote the $n$th $(C, 1)$ means of the sequences $\left(s_{n}\right)$ and $\left(n a_{n}\right)$ by $u_{n}$ and $t_{n}$, respectively. The series $\sum a_{n}$ is said to be summable $|C, 1|_{k}, k \geq 1$, if (see [2])

$$
\sum_{n=1}^{\infty} n^{k-1}\left|u_{n}-u_{n-1}\right|^{k}<\infty
$$

But since $t_{n}=n\left(u_{n}-u_{n-1}\right)$ (see [4]), condition (1.1) can also be written as

$$
\sum_{n=1}^{\infty} \frac{1}{n}\left|t_{n}\right|^{k}<\infty .
$$

Let $\left(p_{n}\right)$ be a sequence of positive real numbers such that

$$
P_{n}=\sum_{v=0}^{n} p_{v} \rightarrow \infty \quad \text { as } n \rightarrow \infty\left(P_{-i}=p_{-i}=0, i \geq 1\right) .
$$

The sequence-to-sequence transformation

$$
w_{n}=\frac{1}{P_{n}} \sum_{v=0}^{n} p_{v} s_{v}
$$

defines the sequence $\left(w_{n}\right)$ of the $\left(\bar{N}, p_{n}\right)$ mean of the sequence $\left(s_{n}\right)$ generated by the sequence of coefficients $\left(p_{n}\right)$ (see [3]). The series $\sum a_{n}$ is said to be summable $\left|\bar{N}, p_{n}\right|_{k}, k \geq 1$, if (see [1])

$$
\sum_{n=1}^{\infty}\left(\frac{P_{n}}{p_{n}}\right)^{k-1}\left|w_{n}-w_{n-1}\right|^{k}<\infty .
$$

Received by the editors November 14, 1990.

1991 Mathematics Subject Classification. Primary 40D15, 40F05, 40G99.

This research was supported by TBAG-C2 (TÜBITAK). 
In the special case when $p_{n}=1$ for all values of $n$ (resp. $\left.k=1\right),\left|\bar{N}, p_{n}\right|_{k}$ summability is the same as $|C, 1|_{k}$ (resp. $\left|\bar{N}, p_{n}\right|$ ) summability.

\section{MazhaR's TheOREM}

Mazhar [5] has established the following theorem for $|C, 1|_{k}$ summability factors of infinite series.

Theorem A. If $\left(X_{n}\right)$ is a positive monotonic nondecreasing sequence such that

$$
\begin{gathered}
\lambda_{m} X_{m}=O(1) \quad \text { as } m \rightarrow \infty, \\
\sum_{n=1}^{m} n X_{n}\left|\Delta^{2} \lambda_{n}\right|=O(1), \\
\sum_{n=1}^{m} \frac{1}{n}\left|t_{n}\right|^{k}=O\left(X_{m}\right) \quad \text { as } m \rightarrow \infty,
\end{gathered}
$$

then the series $\sum a_{n} \lambda_{n}$ is summable $|C, 1|_{k}, k \geq 1$.

\section{MAIN THEOREM}

The aim of this paper is to generalize Theorem $\mathrm{A}$ for $\left|\bar{N}, p_{n}\right|_{k}$ summability in the form of the following theorem.

Theorem. Let $\left(p_{n}\right)$ be a sequence of positive numbers such that

$$
P_{n}=O\left(n p_{n}\right) \text { as } n \rightarrow \infty .
$$

If $\left(X_{n}\right)$ is a positive monotonic nondecreasing sequence such that conditions (2.1)-(2.2) of Theorem $\mathrm{A}$ are satisfied and

$$
\sum_{n=1}^{m} \frac{p_{n}}{P_{n}}\left|t_{n}\right|^{k}=O\left(X_{m}\right) \text { as } m \rightarrow \infty,
$$

then the series $\sum a_{n} \lambda_{n}$ is summable $\left|\bar{N}, p_{n}\right|_{k}, k \geq 1$.

It should be noted that if we take $p_{n}=1$ for all values of $n$, then we get Theorem A. Also it may be noticed that under the conditions of the theorem, we have that

$$
\Delta \lambda_{n} \rightarrow 0 \text { as } n \rightarrow \infty
$$

\section{LEMMA}

We need the following lemma for the proof of our theorem.

Lemma. Under the conditions of the theorem we have that

$$
\begin{gathered}
\sum_{n=1}^{\infty} X_{n}\left|\Delta \lambda_{n}\right|<\infty, \\
n X_{n}\left|\Delta \lambda_{n}\right|=O(1) \text { as } n \rightarrow \infty .
\end{gathered}
$$

Proof. By (3.3), we can write

$$
\Delta \lambda_{n}=\sum_{v=n}^{\infty} \Delta^{2} \lambda_{v} .
$$


Hence,

$$
\begin{aligned}
\sum_{n=1}^{\infty} X_{n}\left|\Delta \lambda_{n}\right| & =\sum_{n=1}^{\infty} X_{n}\left|\sum_{v=n}^{\infty} \Delta^{2} \lambda v\right| \leq \sum_{n=1}^{\infty} X_{n} \sum_{v=n}^{\infty}\left|\Delta^{2} \lambda_{v}\right| \\
& =\sum_{v=1}^{\infty}\left|\Delta^{2} \lambda_{v}\right| \sum_{n=1}^{v} X_{n} \leq \sum_{v=1}^{\infty} v X_{v}\left|\Delta^{2} \lambda_{v}\right|<\infty \quad \text { (by (2.2)). }
\end{aligned}
$$

Since $\left(n X_{n}\right)$ is increasing, we have

$$
\begin{aligned}
n X_{n}\left|\Delta \lambda_{n}\right| & =n X_{n}\left|\sum_{v=n}^{\infty} \Delta^{2} \lambda_{v}\right| \leq n X_{n} \sum_{v=n}^{\infty}\left|\Delta^{2} \lambda_{v}\right| \\
& \leq \sum_{v=n}^{\infty} v X_{v}\left|\Delta^{2} \lambda_{v}\right|<\infty \quad(\text { by }(2.2)) .
\end{aligned}
$$

This complete the proof of the lemma.

\section{Proof OF THE THEOREM}

Let $\left(T_{n}\right)$ be the sequence of $\left(\bar{N}, p_{n}\right)$ means of the series $\sum a_{n} \lambda_{n}$. Then, by definition, we have

$$
T_{n}=\frac{1}{P_{n}} \sum_{v=0}^{n} p_{v} \sum_{r=0}^{v} a_{r} \lambda_{r}=\frac{1}{P_{n}} \sum_{v=0}^{n}\left(P_{n}-P_{v-1}\right) a_{v} \lambda_{v} .
$$

Then, for $n \geq 1$, we get

$$
T_{n}-T_{n-1}=\frac{p_{n}}{P_{n} P_{n-1}} \sum_{v=1}^{n} P_{v-1} a_{v} \lambda_{v}=\frac{p_{n}}{P_{n} P_{n-1}} \sum_{v=1}^{n} \frac{P_{v-1} \lambda_{v}}{v} v a_{v} .
$$

Applying Abel's transformation to the right-hand side of (5.2), we have

$$
\begin{aligned}
T_{n}-T_{n-1}= & \frac{p_{n}}{P_{n} P_{n-1}} \sum_{v=1}^{n-1} \Delta\left(\frac{P_{v-1} \lambda_{v}}{v}\right) \sum_{r=1}^{v} r a_{r}+\frac{p_{n} \lambda_{n}}{n P_{n}} \sum_{v=1}^{n} v a_{v} \\
= & \frac{(n+1) p_{n} t_{n} \lambda_{n}}{n P_{n}}-\frac{p_{n}}{P_{n} P_{n-1}} \sum_{v=1}^{n-1} p_{v} t_{v} \lambda_{v} \frac{v+1}{v} \\
& +\frac{p_{n}}{P_{n} P_{n-1}} \sum_{v=1}^{n-1} P_{v} \Delta \lambda_{v} t_{v} \frac{v+1}{v}+\frac{p_{n}}{P_{n} P_{n-1}} \sum_{v=1}^{n-1} P_{v} \lambda_{v+1} t_{v} \frac{1}{v} \\
= & T_{n, 1}+T_{n, 2}+T_{n, 3}+T_{n, 4}, \quad \text { say. }
\end{aligned}
$$

To complete the proof of the theorem, by Minkowski's inequality, it is sufficient to show that

$$
\sum_{n=1}^{\infty}\left(\frac{P_{n}}{p_{n}}\right)^{k-1}\left|T_{n, r}\right|^{k}<\infty, \quad \text { for } r=1,2,3,4
$$


74

HÜSEYIN BOR

First, we have

$$
\begin{aligned}
\sum_{n=1}^{m}\left(\frac{P_{n}}{p_{n}}\right)^{k-1}\left|T_{n, 1}\right|^{k} & =O(1) \sum_{n=1}^{m}\left|\lambda_{n}\right|^{k-1}\left|\lambda_{n}\right| \frac{p_{n}}{P_{n}}\left|t_{n}\right|^{k}=O(1) \sum_{n=1}^{m}\left|\lambda_{n}\right| \frac{p_{n}}{P_{n}}\left|t_{n}\right|^{k} \\
& =O(1) \sum_{n=1}^{m-1} \Delta\left|\lambda_{n}\right| \sum_{v=1}^{n} \frac{p_{v}}{P_{v}}\left|t_{v}\right|^{k}+O(1)\left|\lambda_{m}\right| \sum_{v=1}^{m} \frac{p_{v}}{P_{v}}\left|t_{v}\right|^{k} \\
& =O(1) \sum_{n=1}^{m-1}\left|\Delta \lambda_{n}\right| X_{n}+O(1)\left|\lambda_{m}\right| X_{m}=O(1) \quad \text { as } m \rightarrow \infty,
\end{aligned}
$$

by virtue of the hypotheses and Lemma.

Now, applying Hölder's inequality, as in $T_{n, 1}$, we have that

$$
\begin{aligned}
& \sum_{n=2}^{m+1}\left(\frac{P_{n}}{p_{n}}\right)^{k-1}\left|T_{n, 2}\right|^{k}=O(1) \sum_{n=2}^{m+1} \frac{p_{n}}{P_{n} P_{n-1}}\left\{\sum_{v=1}^{n-1} p_{v}\left|t_{v}\right|^{k}\left|\lambda_{v}\right|^{k}\right\} \times\left\{\frac{1}{P_{n-1}} \sum_{v=1}^{n-1} p_{v}\right\}^{k-1} \\
& \quad=O(1) \sum_{v=1}^{m}\left|\lambda_{v}\right|^{k-1}\left|\lambda_{v}\right| p_{v}\left|t_{v}\right|^{k} \sum_{n=v+1}^{m+1} \frac{p_{n}}{P_{n} P_{n-1}} \\
& \quad=O(1) \sum_{v=1}^{m}\left|\lambda_{v}\right| \frac{p_{v}}{P_{v}}\left|t_{v}\right|^{k}=O(1) \text { as } m \rightarrow \infty .
\end{aligned}
$$

Again, using the fact that $P_{v}=O\left(v p_{v}\right)$, by (3.1), we get

$$
\begin{aligned}
\sum_{n=2}^{m+1}\left(\frac{P_{n}}{p_{n}}\right)^{k-1}\left|T_{n, 3}\right|^{k} & \\
= & O(1) \sum_{n=2}^{m+1} \frac{p_{n}}{P_{n} P_{n-1}^{k}}\left\{\sum_{v=1}^{n-1} P_{v}\left|\Delta \lambda_{v}\right|\left|t_{v}\right|\right\}^{k} \\
& =O(1) \sum_{n=2}^{m+1} \frac{p_{n}}{P_{n} P_{n-1}^{k}}\left\{\sum_{v=1}^{n-1} v p_{v}\left|\Delta \lambda_{v}\right|\left|t_{v}\right|\right\}^{k} \\
& =O(1) \sum_{n=2}^{m+1} \frac{p_{n}}{P_{n} P_{n-1}}\left\{\sum_{v=1}^{n-1}\left(v\left|\Delta \lambda_{v}\right|\right)^{k} p_{v}\left|t_{v}\right|^{k}\right\} \times\left\{\frac{1}{P_{n-1}} \sum_{v=1}^{n-1} p_{v}\right\}^{k-1} \\
& =O(1) \sum_{v=1}^{m}\left(v\left|\Delta \lambda_{v}\right|\right)^{k-1} v\left|\Delta \lambda_{v}\right| p_{v}\left|t_{v}\right|^{k} \sum_{n=v+1}^{m+1} \frac{p_{n}}{P_{n} P_{n-1}} \cdot \\
& =O(1) \sum_{v=1}^{m} v\left|\Delta \lambda_{v}\right| \frac{p_{v}}{P_{v}}\left|t_{v}\right|^{k} \\
& =O(1) \sum_{v=1}^{m-1} \Delta\left(v\left|\Delta \lambda_{v}\right|\right) \sum_{r=1}^{v} \frac{p_{r}}{P_{r}}\left|t_{r}\right|^{k}+O(1) m\left|\Delta \lambda_{m}\right| \sum_{v=1}^{m} \frac{p_{v}}{P_{v}}\left|t_{v}\right|^{k}
\end{aligned}
$$




$$
\begin{aligned}
& =O(1) \sum_{v=1}^{m-1}\left|\Delta\left(v\left|\Delta \lambda_{v}\right|\right)\right| X_{v}+O(1) m\left|\Delta \lambda_{m}\right| X_{m} \\
& =O(1) \sum_{v=1}^{m-1} v X_{v}\left|\Delta^{2} \lambda_{v}\right|+O(1) \sum_{v=1}^{m-1}\left|\Delta \lambda_{v+1}\right| X_{v}+O(1) m\left|\Delta \lambda_{m}\right| X_{m} \\
& =O(1) \text { as } m \rightarrow \infty,
\end{aligned}
$$

by virtue of the hypotheses and Lemma.

Finally, using the fact that $P_{v}=O\left(v p_{v}\right)$, by $(3.1)$, as in $T_{n, 1}$, we have that

$$
\begin{aligned}
& \sum_{n=1}^{m+1}\left(\frac{P_{n}}{p_{n}}\right)^{k-1}\left|T_{n, 4}\right|^{k}=O(1) \sum_{n=2}^{m+1} \frac{p_{n}}{P_{n} P_{n-1}^{k}}\left\{\sum_{v=1}^{n-1}\left|\lambda_{v+1}\right| p_{v}\left|t_{v}\right|\right\}^{k} \\
& \quad=O(1) \sum_{n=2}^{m+1} \frac{p_{n}}{P_{n} P_{n-1}}\left\{\sum_{v=1}^{n-1}\left|\lambda_{v+1}\right|^{k} p_{v}\left|t_{v}\right|^{k}\right\} \times\left\{\frac{1}{P_{n-1}} \sum_{v=1}^{n-1} p_{v}\right\}^{k-1} \\
& \quad=O(1) \sum_{v=1}^{m}\left|\lambda_{v+1}\right|^{k-1}\left|\lambda_{v+1}\right| p_{v}\left|t_{v}\right|^{k} \sum_{n=v+1}^{m+1} \frac{p_{n}}{P_{n} P_{n-1}} \\
& \quad=O(1) \sum_{v=1}^{m}\left|\lambda_{v+1}\right| \frac{p_{v}}{P_{v}}\left|t_{v}\right|^{k}=O(1) \text { as } m \rightarrow \infty
\end{aligned}
$$

Therefore, we get that

$$
\sum_{n=1}^{m}\left(\frac{P_{n}}{p_{n}}\right)^{k-1}\left|T_{n, r}\right|^{k}=O(1) \quad \text { as } m \rightarrow \infty, \text { for } r=1,2,3,4 \text {. }
$$

This completes the proof of the theorem.

\section{REFERENCES}

1. H. Bor, On two summability methods, Math. Proc. Cambridge Philos. Soc. 97 (1985), 147149.

2. T. M. Flett, On an extension of absolute summability and some theorems of Littlewood and Paley, Proc. London Math. Soc. 7 (1957), 113-141.

3. G. H. Hardy, Divergent series, Oxford Univ. Press, New York and London, 1949.

4. E. Kogbetliantz, Sur les séries absolument sommables par la méthode des moyennes arithmétiques, Bull. Sci. Mat. 49 (1925), 234-256.

5. S. M. Mazhar, On $|C, 1|_{k}$ summability factors of infinite series, Indian J. Math. 14 (1972), 45-48.

Department of Mathematics, Erciyes University, Kaysert 38039, Turkey

Mailing address: P.K. 213, Kayseri 38002, Turkey

E-mail address: BOR@TRERUN.BITNET 